\title{
¿Es relevante incorporar la medición de la mortalidad en los censos de América Latina y el Caribe?
}

\author{
Bernardo L. Queiroz \\ Nicolás Sacco
}

\section{Introducción}

Dentro del combo de sugerencias de las Naciones Unidas para los censos de población, se encuentran aquellas propuestas que remiten a la inclusión de la dimensión de mortalidad, tanto infantil como también adulta. En resumidas cuentas, dos argumentos contrapuestos se utilizan en torno a la incorporación, o no, de su medición en las fuentes censales. Por un lado, los que señalan que únicamente deberían emplearse en aquellos países que cuentan con estadísticas vitales de dudosa calidad y/o de poca cobertura; por el otro, en cambio, los que sostienen que su introducción en los censos de población permitiría la ampliación del conocimiento acumulado sobre mortalidad que se deriva de las estadísticas vitales, independientemente de su cobertura y/o calidad, gracias a un mayor número de características socio-económicas relevadas en simultáneo y la amplia cobertura geográfica.

Centrándonos en la medición de la mortalidad para todas las edades en base a datos de registros vitales y censales, el objetivo de esta comunicación es ampliar los argumentos, tanto a favor como en contra, de estos puntos de vista, mostrar la experiencia reciente al respecto en América Latina y el Caribe para, por último, proponer recomendaciones en base al análisis realizado, con el foco puesto en la próxima ronda censal.

Se hace énfasis en Latinoamérica, región de heterodoxa experiencia, ya que en su interior conviven situaciones diferenciales en cuanto a disponibilidad y calidad de los datos para el estudio de la mortalidad. En términos generales, conviven dos grupos: por un lado, aquellos países que cuentan, en al menos una de las tres últimas rondas censales, con la medición de las muertes en el hogar (por ejemplo, Brasil) y que adicionalmente tienen un sistema de estadísticas vitales de regular y/o buena calidad; por el otro, aquellos que históricamente poseen un sistema de registro de vitales de muy buena calidad (por ejemplo, Argentina) pero cuyos censos de población nunca preguntaron por las muertes en el hogar.

\section{Motivaciones para incluir la pregunta en los censos}

La inclusión de la información sobre las muertes en el hogar en los últimos 12 meses forma parte de los Principios y Recomendaciones para los Censos de Población y Vivienda de las Naciones Unidas (ONU, 1998) para la ronda de censos de 2000 y 2010. La última versión de este manual, propuesta para el 2020, sugiere que esta pregunta debería hacerse en aquellos países que no cuentan con un sistema de estadísticas de muerte satisfactoria (ONU, 2015: 234). Los datos derivados de tal información pueden ayudar a evaluar las tendencias en los niveles, y algunas causas, de la mortalidad, tanto adulta, como de todas las edades. El uso de estas simples preguntas debería permitir derivar información sobre las principales tendencias en mortalidad que, de otro modo, utilizando otra fuente, como las vitales, serían difíciles de obtener (ONU, 2015 ). 
Es que, en efecto, las estadísticas de registro representan la fuente más coherente y directa para obtener estimaciones de mortalidad por edad en buena parte de los países de la región. Sin embargo, algunos países de América Latina y el Caribe están afectados por un fuerte subregistro de muertes (Luy, 2011; Palloni y Pinto-Aguirre, 2011). De esta forma, en el caso de adicionar temas relativos a salud, la Organización Mundial de la Salud (OMS), por ejemplo, sugiere incluir un número breve de preguntas en los censos de población que pueden ser usados, a su vez, para generar estimativas de cobertura de los registros vitales (WHO, 2014).

Según la División de Población de Naciones Unidas (ONU, 2014), sólo 12 países de América Latina y el Caribe poseen una cobertura de muertes menor al $90 \%$. Para este conjunto, las estimaciones de mortalidad son limitadas de acuerdo a vitales y afectan todo el sistema de planificación de salud de los países y sus regiones. En un contexto más amplio, a la vez, perjudicadas por estas limitaciones, esta situación afecta aquellos avances que se pueden haber dado en las condiciones de salud y en la consecución de las Metas del Milenio, propuestas por las Naciones Unidas. En esos casos, por ende, el censo se posiciona como la fuente principal para contar con estimaciones de mortalidad de población.

\section{¿Cómo podemos estudiar la mortalidad con los datos que tenemos? La experiencia regional}

Si los datos del registro civil son de buena cobertura y calidad, la mortalidad se puede estimar desde esa fuente y, de hecho, esta constituye la práctica habitual de la mayor parte de los países de la región. En los casos que no lo son, siguiendo recomendaciones de la ONU, diversos países incluyeron preguntas relacionadas a la mortalidad en los censos. A través de la consulta sobre la ocurrencia de muerte de algún miembro del hogar en los últimos 12 meses y el sexo y la edad del fallecido ${ }^{1}$, mediante la aplicación de métodos demográficos es posible derivar medidas tales como la esperanza de vida al nacer en base a esta fuente.

La Tabla 1 muestra aquellos países de la región que incorporaron preguntas relativas a las muertes en el hogar en alguna de las últimas tres rondas censales. Se pueden distinguir dos grupos: i) aquellos que se caracterizan por una baja cobertura de estadísticas vitales (menores al 90\%) (como, por ejemplo, Bolivia y El Salvador) e incluyeron esta dimensión en los censos y ii) aquellos países que cuentan con una satisfactoria cobertura de estadísticas vitales (superior al 90\%) pero han incorporado a su vez, la indagación de muertes en el hogar en alguna ronda censal (por ejemplo, Brasil y Colombia).

\footnotetext{
${ }^{1}$ Para el caso de muertes de sexo femenino, algunos países incluyeron preguntas que permiten identificar si esa muerte estuvo relacionada con el embarazo.
} 
Tabla 1. País de América Latina y el Caribe que incorporaron preguntas relativas a la mortalidad en el hogar, según ronda y año.

\begin{tabular}{|c|c|c|c|}
\hline \multirow{2}{*}{ País } & \multicolumn{3}{|c|}{ Ronda y Año } \\
\cline { 2 - 4 } & $\mathbf{1 9 9 0}$ & $\mathbf{2 0 0 0}$ & $\mathbf{2 0 1 0}$ \\
\hline Bolivia & 1992 & 2001 & \\
Brasil & & & 2010 \\
Colombia & & 2005 & \\
Costa Rica & & & \\
El Salvador & 1992 & & 2007 \\
Guatemala & 1994 & & \\
Haití & 1982 & 2003 & \\
Honduras & 1988 & 2001 & \\
Nicaragua & 1995 & 2005 & \\
Panamá & & 2000 & \\
Paraguay & & 2002 & 2012 \\
República Dominicana & & 2002 & 2010
\end{tabular}

Fuente: elaboración propia en base a censos de población.

En cualquier de estos casos, los censos demográficos permiten obtener distintos tipos de estimaciones de mortalidad: más adecuadas en los países con baja cobertura de vitales, complementarias en el caso de los países con un buen sistema de registro.

A pesar de sus ventajas relativas, sobre todo en lo casos donde no se cuenta con otro tipo de información, el censo como fuente subsidiaria a las necesidades de información es también limitado para indagar en profundidad sobre aspectos complejos, en particular por lo que respecta al espacio posible para incluir preguntas en el cuestionario y la posibilidad de no contar con censistas entrenados o informantes calificados dentro del hogar. En líneas generales, los datos de muerte en el hogar deben ser evaluados con cuidado pues están sujetos a cuatro problemas principales:

1) sub-enumeración de muertes debido: i) a disolución de domicilios después de la muerte de un residente o a ii) una alta concentración de población en hogares unipersonales y/o iii) desintegración de hogares compuestos por adultos mayores;

2) jóvenes que migran de sus domicilios de origen para poder ser registrados como pertenecientes a más de un domicilio (lo que puede dar lugar a la sobre-estimación de muertes entre los jóvenes); 
3) errores en: periodo de referencia; concepto de mortalidad en el último año (calendario); problemas en el diseño de la cédula censal; problemas en la declaración de sexo y edad, cantidad de casos; definición de hogar/familia.

4) posibilidad de fluctuaciones aleatorias y significativas de mortalidad que afectan las estimativas de interés.

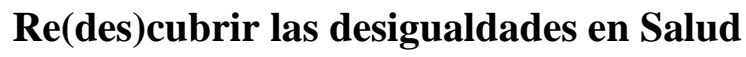

Entonces, ¿los institutos de estadística de la región (muchos de los cuáles se encuentran en pleno desarrollo de los preparativos para la próxima ronda censal) deben desistir de incorporar la pregunta de muertes en el hogar en los últimos 12 meses en los censos cuando los datos de registro son de buena calidad y cobertura? Por lo señalado previamente, el argumento central para su exclusión es que el que registro de muertes en el censo no es necesario, ya que se cuenta con esa información en las estadísticas de registro. A la vez, la información censal releva, en todo caso, información de peor calidad que las vitales (Hill y Stanton, 2011; Queiroz y Sawyer, 2012).

Sin embargo, surgen varios interrogantes sobre estos argumentos. En primer lugar: ¿cómo saber que tan buenos los datos de vitales son? Si bien se conoce una mejora significativa en los últimos 30 años en las estadísticas de registro de cada uno de los países de la región (Luy, 2011), a nivel total, todavía es poco lo que sabe sobre los diferenciales al interior de cada país. En Brasil, por ejemplo, la cobertura de estadísticas vitales pasó del 80\% al 95\% entre fines del siglo XX y principios del siglo XXI. Pero un gran diferencial regional todavía subyace entre las regiones desarrolladas del Sur y las del Norte (Paes y Albuquerque, 1999; Paes, 2005; Queiroz y otros, 2013; Lima y Queiroz, 2014 ; Freire y otros, 2015; Queiroz y otros, 2017).

Se puede sospechar que lo mismo sucede en el resto de los países que cuentan con altos niveles de cobertura: supóngase un país como Argentina, con muy buena calidad de vitales: a nivel total país esto puede ser cierto, pero es bien conocido que la heterogeneidad entre las provincias es considerable, tanto en su cobertura como en la definición de causas de muerte. Los problemas de calidad de los datos relativos a las muertes es posible que se den con mayor frecuencia en localidades o áreas con menores niveles de desarrollo económico, $\mathrm{y}$ con mayores desigualdades sociales y en salud.

En segundo lugar, admitiendo que la cobertura y la calidad de las vitales son buenas a lo largo y ancho de las regiones de cada país, si se comparan con registros vitales completos y de calidad (o con aquellos que presentan avances significativos), las preguntas adicionales sobre mortalidad son muy limitadas en los registros. La gran ventaja del censo es que permite la construcción de información a niveles sub-nacionales y sub-provinciales y el procesamiento de datos a nivel desagregado, con el cruce de variables no disponibles en los registros de vitales, tales como, por ejemplo, la ocupación. Con estos datos, es factible estudiar diferenciales sociales de mortalidad, poco viable con los datos de las estadísticas vitales. 
$\mathrm{Y}$ es que, de hecho, existe una amplia literatura sobre la relación entre las desigualdades sociales y las condiciones de salud y mortalidad. La evidencia empírica en distintos países, a nivel total país, pero también a nivel de áreas menores, sugiere la existencia de una asociación positiva entre los niveles de desigualdad social e indicadores agregados de salud y mortalidad. Pero el punto principal no es solamente ese: la pregunta en el censo da lugar una serie de estudios para áreas menores y cruces con otras variables, pero también su información derivada permite evaluar la calidad de los datos de registro de muertes.

En la Tabla 2, a modo de ejemplo, se enumeran algunas de las publicaciones más recientes y relevantes que han utilizado datos censales para estudiar la mortalidad:

Tabla 2. Principales publicaciones que utilizaron información censal para realizar estudios relativos a la mortalidad, según Autores (y año), País de estudios y Tema principal.

\begin{tabular}{|c|c|c|}
\hline Autores & País de estudio & Temas principales \\
\hline Queiroz y Sawyer (2012) & Brasil & $\begin{array}{l}\text { Evaluación de la calidad de los } \\
\text { datos censales. Resultados } \\
\text { muy robustos }\end{array}$ \\
\hline Silva, Freire y Pereira (2016) & Brasil y regiones & $\begin{array}{l}\text { Mortalidad diferencial por } \\
\text { niveles educacionales y } \\
\text { regiones }\end{array}$ \\
\hline Queiroz (2011) & Honduras & $\begin{array}{l}\text { Diferencias regionales en } \\
\text { mortalidad materna }\end{array}$ \\
\hline Hill et. al. (2009) & $\begin{array}{l}\text { Honduras, Paraguay y } \\
\text { Nicaragua }\end{array}$ & Mortalidad materna \\
\hline Leone (2014) & $\begin{array}{l}\text { Honduras, Paraguay y } \\
\text { Nicaragua }\end{array}$ & Mortalidad materna \\
\hline Ribeiro, Turra y Pinto (2016) & Brasil & $\begin{array}{l}\text { Diferenciales educacionales en } \\
\text { mortalidad adulta }\end{array}$ \\
\hline Pereira y Queiroz (2016) & Brasil y municipios & $\begin{array}{l}\text { Diferencias socio-económicas } \\
\text { en la mortalidad de jóvenes } \\
\text { adultos }\end{array}$ \\
\hline $\begin{array}{l}\text { Barbosa, Mendes, Queiroz y } \\
\text { Ventura (2017) }\end{array}$ & Brasil & $\begin{array}{l}\text { Diferenciales de mortalidad } \\
\text { por grupos étnicos }\end{array}$ \\
\hline $\begin{array}{l}\text { Castro, Fajnzylber, Fortunato } \\
\text { (2017) }\end{array}$ & Brasil y El Salvador & $\begin{array}{l}\text { Diferenciales de mortalidad } \\
\text { por grupos socio-económicos }\end{array}$ \\
\hline Brizuela (2005) & Paraguay & $\begin{array}{l}\text { Diferenciales de mortalidad } \\
\text { para áreas menores }\end{array}$ \\
\hline
\end{tabular}

Fuente: elaboración propia en base a censos de población. 


\section{Conclusión}

El continuo desarrollo de los sistemas de registro de estadísticas vitales de muertes y nacimientos sigue siendo una tarea con muchos desafíos para mayor de los países de América Latina. En un mundo donde se debate realizar censos continuos en base a registros vitales, no contar con un sistema plenamente satisfactorio de hechos vitales básicos, como la muerte y el nacimiento, constituye un gran desafío para los sistemas de información en salud de cada país. La información derivada de un continuo mejoramiento de esta información permitiría contar con una base aún más sólida para la elaboración de proyecciones de población (ya que es su insumo básico), sobre todo para cubrir las necesidades de información a nivel sub-áreas, indispensables para la planificación económica y social. En este sentido, contar con datos sobre muerte en los censos de población constituye un marco básico para el control de calidad de las vitales.

En fin, los datos censales todavía ofrecen una excelente oportunidad para el estudio de diferenciales regionales y/o socioeconómicos de mortalidad, más complejos de abordar con otras fuentes. Los datos de mortalidad derivados de los censos poseen dos importantes ventajas: abren una gran oportunidad para estudios de mortalidad en pequeñas áreas y según diferenciales socio-económicos y sus medidas resumen utilizan numeradores y denominadores de la misma fuente. A la vez, la inclusión de estas informaciones en los censos, debido a su bajo costo y facilidad de aplicación, son una fuente de importante de información, no sólo en el caso de países menos desarrollados o en desarrollo, que no cuentan con un sistema de registro adecuado. 


\section{Bibliografía}

Brizuela de Ramírez, F. (2005). Mortalidad: evolución y tendencias : 1970-2000 Serie Condiciones de vida en Paraguay: Dirección General de Estadística, Encuestas y Censos.

Campos, M. B. d., Borges, G. M., Queiroz, B. L., y Santos, R. V. (2017). "Diferenciais de mortalidade entre indígenas e não indígenas no Brasil com base no Censo Demográfico de 2010". Cadernos de Saúde Pública, 33. doi: http://dx.doi.org/10.1590/10.1590/0102-311x00015017.

Castro, R., Fajnzylber, E., y Fortunato, A. (2017). "Inequality in mortality decreases with age: Evidence from developing countries using census data". Canadian Studies in Population, 44(3-4), 125-133. doi: http://dx.doi.org/10.25336/P60G7G.

Freire, F. H. M. d. A., Queiroz, B. L., Lima, E. E. C. d., Gonzaga, M. R., y Souza, F. H. (2015). Mortality Estimates and Construction of Life Tables for Small Areas in Brazil, 2010. Ponencia presentada en Population Association of America 2015 Anual Meeting, San Diego, CA.

Hill, K., Queiroz, B. L., Wong, L., Plata, J., Popolo, F. D., Rosales, J., y Stanton, C. (2009). "Estimating pregnancy-related mortality from census data: experience in Latin America". Bulletin of the World Health Organization, 87(4 ), 288-295.

Hill, K., y Stanton, C. (2011). "Measuring maternal mortality through the census: rapier or bludgeon?". Journal of Population Research, 28(1 ), 31-47.

Leone, T. (2014). "Measuring differential maternal mortality using census data in developing countries". Population, Space and Place, 20(7), 581-591.

Lima, E. E. C. d., y Queiroz, B. L. (2014 ). "Evolution of the deaths registry system in Brazil: associations with changes in the mortality profile, under-registration of death counts, and ill-defined causes of death". Cadernos de Saúde Pública, $30(8$ ), 17211730. doi: https://dx.doi.org/10.1590/0102-311X00131113.

Luy, M. (2011). "A classification of the nature of mortality data underlying the estimates for the 2004 and 2006 United Nations' World Population Prospects". Comparative Population Studies, 35(2).

ONU. (1998). Principios y recomendaciones para los censos de población y habitación. Revisión 1. Nueva York: Naciones Unidas.

---. (2014). World Population Prospects. The 2012 Revision. Methodology of the United Nations Population Estimates and Projections. Working Paper No. ESA/P/WP.23. Department of Economic and Social Affairs, Population Division. United Nations. New York.

---. (2015). Principles and Recommendations for Population and Housing Censuses. Revision 3 (Vol. Series M. No. 67/Rev.3).

Paes, N. A. (2005). "Avaliação da cobertura dos registros de óbitos dos Estados brasileiras em 2000". Revista de Saúde Pública, 39(6), 882-890.

Paes, N. A., y Albuquerque, M. E. E. (1999). "Avaliação da qualidade dos dados populacionais e cobertura dos registros de óbitos para as regiões brasileiras". Revista de Saúde Pública, 33(1). doi: http://dx.doi.org/10.1590/S003489101999000100006. 
Palloni, A., y Pinto-Aguirre, G. (2011). Adult mortality in Latin America and the Caribbean. En R. G. Rogers y E. M. Crimmins (Eds.), International Handbook of Adult Mortality (pp. 101-132): Springer Netherlands.

Pereira, F. N. A., y Queiroz, B. L. (2016). "Differences in mortality in Brazilian youth: the importance of household socioeconomic factors and living conditions in Brazilian municipalities and states". Cadernos de Saúde Pública, 32(9).

Queiroz, B. L. (2011). "Estimating maternal mortality differentials using census data: experience in Honduras". Journal of Population Research, 1(1), 75-87.

Queiroz, B. L., Freire, F. H. M. d. A., Gonzaga, M. R., y Lima, E. E. C. d. (2017). "Completeness of death-count coverage and adult mortality (45q15) for Brazilian states from 1980 to 2010". Revista Brasileira de Epidemiologia 20(Suppl. 1), 21-33. doi: https://dx.doi.org/10.1590/1980-54972017000500033

Queiroz, B. L., Lima, E. E. C. d., Freire, F. H. M. d. A., y Gonzaga, M. R. (2013). "Adult mortality estimates for small areas in Brazil, 1980-2010: a methodological approach". Lancet (British edition), 381, 120-120.

Queiroz, B. L., y Sawyer, D. O. (2012). "O que os dados de mortalidade do Censo de 2010 podem nos dizer?". Revista Brasileira de Estudos de População, 29(2), 225-238.

Ribeiro, M. M., Turra, C. M., y Pinto, C. C. X. (2016). Estimativas de mortalidade adulta por níveis de escolaridade no estado São Paulo em 2010. Ponencia presentada en XX Encontro Nacional de Estudos Populacionais, Foz do Iguaçu.

Silva, L. E. d., Freire, F. H. M. d. A., y Pereira, R. H. M. (2016). "Diferenciais de mortalidade por escolaridade da população adulta brasileira, em 2010". Cadernos de Saúde Pública, 32(4).

WHO. (2014). Improving Mortality Statistics through Civil Registration and Vital Statistics Systems Strategies for country and partner support. Geneva. 